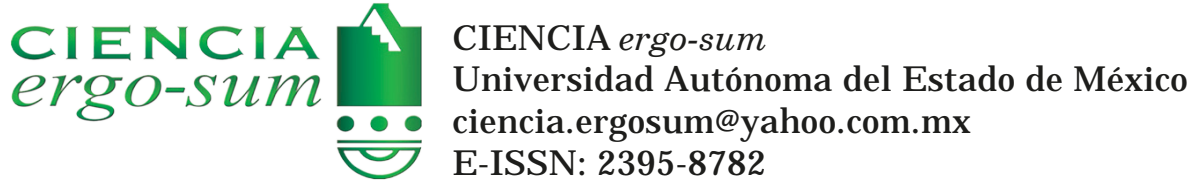

\title{
Modelos Lagrangianos para la simulación de fluidos y su aplicación a la hidrodinámica marina
}

Sánchez-Mondragón, J oel

Model os Lagrangianos para la simulación de fluidos y su aplicación a la hidrodinámica marina

CIENCIA ergo-sum, Número especial "Retos de la física no lineal" 2020|e105

Universidad Autónoma del Estado de México, México

Esta obra está bajo una Licencia Creative Commons Atribución-NoComercial-SinDerivar 4.0 Internacional .

Sánchez-Mondragón, J . (2020). Modelos Lagrangianos para la simulación de fluidos y su aplicación a la hidrodinámica marina. CIE NCIA ergo-sum, Número especial "Retos de la física no lineal". 


\title{
Modelos Lagrangianos para la simulación de fluidos y su aplicación a la hidrodinámica marina
}

Lagrangian models for the simulation of fluids and their application to marine hydrodynamics

Joel Sánchez-Mondragón

CONACYT Research Fellow-Instituto Mexicano del Petróleo, México

Recepción: 24 de junio de 2019

jsmbalam@gmail.com

Aprobación: 10 de febrero de 2020

(D) http://orcid.org/0000-0002-9875-7380

\begin{abstract}
Resumen
Se describe el método de movimiento de partículas semi-implícito (MPS), el cual es uno de los modelos Lagrangianos más representativos usados en la simulación de fluidos. Además, se especifican las ventajas y desventajas de las versiones del método MPS: una basada en la densidad de partículas, otra en la divergencia de la velocidad y la débilmente compresible. Adicionalmente, para demostrar la capacidad del método, estas versiones se aplican en el problema clásico de rompimiento de presa en dos dimensiones. De estos resultados, se comparan las distribuciones del campo de presiones en varias etapas de la simulación y el histórico de la presión de impacto en la pared del contenedor por la colisión con el fluido.
\end{abstract}

Palabras ClaVe: modelos Lagrangianos, simulación de fluidos, método de movimiento de partículas semi-implícito.

\section{Abstract}

The Semi-Implicit Particle Movement (MPS) method, which is one of the most representative models of the Lagrangian models used in fluid simulation, is described in this work. Moreover, advantages and disadvantages of the MPS method are described: from the version based on the particle number density, the velocity divergence and the weakly compressible versions. Furthermore, to demonstrate the method capabilities, these versions are compared on the basis of the classical two dimensional dam-breaking problem. From these results, the differences on the pressure field distribution on several time steps and the pressure time histories on the wall impact zone are analyzed.

KEYWORDS: Lagrangian models, fluid simulation, semi-implicit particle movement method.

\section{INTRODUCCIÓN}

El estudio de la hidrodinámica tiene un amplio rango de aplicaciones que va desde el movimiento de la sangre en las venas hasta la formación de fenómenos naturales como huracanes y ciclones. Estos fenómenos constituyen un reto para su descripción debido a su alta no linealidad, a los efectos turbulentos que deben tomarse en consideración y, en algunos casos, a la naturaleza multifásica del problema a estudiar. Para analizar el comportamiento de estos fluidos pueden usarse métodos analíticos, numéricos o a través de experimentos. Cada uno de estos enfoques proporciona ventajas y desventajas sobre las demás aproximaciones dependiendo de la complejidad del fenómeno. En algunos casos se pueden obtener soluciones analíticas para las cuales comúnmente es necesario suponer simplificaciones como las que representan geometrías más elementales o suposiciones que reduzcan las ecuaciones para su solución (por ejemplo una aproximación lineal). Una alternativa a las dificultades presentadas por las soluciones analíticas es la representada por los métodos numéricos, los cuales hacen aproximaciones sobre las expresiones analíticas a través de discretizaciones espaciales y temporales reduciendo su precisión con respecto a las soluciones analíticas. De esta forma, los métodos numéricos permiten resolver los problemas en escenarios reales e incluso plantear escenarios hipotéticos más complejos que aquellos que pueden resolverse de forma analítica. Sin embargo, una de las limitantes de los métodos numéricos es el costo computacional, pues entre mayor 
sea el dominio o refinamiento de la solución mayor será la cantidad de cálculos que se requieren. Por último, se pueden desarrollar estudios experimentales o mediciones en sitio, los cuales van a proporcionar datos reales y precisos; la limitante que se tiene en este caso es el costo de las pruebas, el cual suele ser significativamente alto en comparación con las soluciones analíticas y numéricas.

Con las consideraciones anteriores, el desarrollo de pruebas numéricas se ha convertido en una alternativa factible y de bajo costo para el estudio de fenómenos hidrodinámicos con múltiples aplicaciones. Los métodos numéricos aplicados a problemas de hidrodinámica se pueden clasificar en dos grandes grupos: los métodos Eulerianos y los métodos Lagrangianos. Los métodos Eulerianos requieren la discretización del medio continuo a través del uso de mallas, las cuales son estructuras geométricas que contienen puntos o nodos donde se resuelven las ecuaciones que gobiernan dicho sistema. Por otro lado, los métodos Lagrangianos no requieren el uso mallas, ya que consideran la concentración de las propiedades del fluido en puntos materiales llamados partículas, por lo cual también se les conoce como métodos de partículas. Una de las ventajas de los métodos Lagrangianos es que pueden analizar fácilmente superficies libres sin difusión numérica debido a que se sigue el movimiento de cada partícula del fluido a lo largo de toda la simulación sin el uso de términos convectivos. La descripción del fluido sin usar mallas de los métodos de partículas proporciona una de las propiedades más destacables, pues permite modelar fenómenos en hidrodinámica marina tan complejos como el comportamiento de oleaje extremo, en donde la fragmentación y coalescencia representan un reto para los métodos basados en mallas. Además, otra ventaja es la representación de la interacción fluido-estructura que requiere un proceso de remallado cuando el sólido se mueve en el esquema Euleriano, mientras que en el esquema Lagrangiano basta con representar la estructura a través de partículas con simples restricciones, como su movimiento respecto al centro de masa, entre otros. Otra cualidad importante es la distribución suavizada de las propiedades de las partículas que se realiza usando una función núcleo para promediar las cantidades físicas sobre las partículas vecinas. En contraste otros métodos Eulerianos, como el método de diferencias finitas, no siempre son numéricamente estables durante la fase de fragmentación dadas las restricciones del mallado sobre el dominio simulado, como ya se mencionó.

Los métodos de partículas pueden clasificarse en $a$ ) los basados en aproximaciones de campo, como el método de elemento libre de Galerkin (Element Free Galerkin), y $b$ ) los basados en aproximaciones de la función núcleo o kernel, como los métodos de hidrodinámica suavizada de partículas (SPH: Smoothed Particle Hydrodynamics) y el método de movimiento de partículas semi-implícito (MPS: Moving Particle Semi-implicit). Ambos métodos, SPH y MPS, utilizan partículas para la discretización del fluido y calculan su comportamiento basado en la ecuación de conservación de masa y en la ecuación de conservación de momentum; sin embargo, su idea básica es diferente: el método SPH considera que las cantidades físicas de una partícula, como la densidad de masa o la velocidad y la presión, no pertenecen a la partícula, pero considera que se distribuyen suavemente alrededor de ella, y la función núcleo se utiliza para calcular la distribución de cantidades físicas. Por otro lado, el método MPS considera que las cantidades físicas como la densidad de masa o velocidad pertenecen a la partícula y calcula la interacción entre partículas con la función núcleo.

El método SPH fue desarrollado para resolver problemas en astrofísica por Lucy (1977) y ha sido extendido al estudio de la dinámica de fluidos por Gingold y Monaghan (1977), considerando en su formulación original una aproximación débilmente incompresible. El método MPS fue desarrollado por Koshizuka y Oka (1996) y Koshizuka et al. (1998) y su principal característica es que emplea un algoritmo semi-implícito para el acoplamiento entre la presión y los componentes de la velocidad de un fluido incompresible. Los métodos SPH y MPS son usados comúnmente para la simulación de fenómenos en fluidos con un comportamiento altamente no lineal como la fragmentación y coalescencia del fluido (Koshizuka et al., 1998; Shao y Lo, 2003).

En los métodos SPH y MPS se define una función de interacción entre partículas por medio de la cual se distribuyen las propiedades físicas del fluido (Gingold y Monaghan, 1977; Koshizuka et al., 1998). Esta función núcleo depende de un radio de interacción permitiendo delimitar la interacción a los vecinos más cercanos a cada 
partícula y reduce a su vez el tiempo de cómputo. Los elementos computacionales en los métodos de SPH y MPS son por consiguiente un número discreto de partículas del fluido con una distribución arbitraria determinada por su posición y que son seguidas en el tiempo usando la velocidad de cada partícula en cada instante de tiempo.

Los métodos SPH y MPS se desarrollan considerando el cambio de posición de cada partícula determinado a través de la interacción con las partículas vecinas resolviendo las ecuaciones gobernantes a través de los modelos de interacción entre partículas, que están descritos a su vez por los siguientes operadores diferenciales: el gradiente, la divergencia, el Laplaciano y un modelo de superficie libre, que es considerado como condición de frontera. Para satisfacer la ecuación de conservación de masa, en el caso de los fluidos incompresibles, la densidad del fluido se considera como constante. Usando esta condición, la presión se calcula en cada instante de tiempo de forma implícita al resolver la ecuación de Poisson de la presión, mientras que los otros términos son calculados explícitamente. Para el caso de los fluidos con una compresibilidad "débil" se pueden usar otras aproximaciones como son las funciones de estado para el cálculo de la presión o factores de relajación introducidos en la ecuación de Poisson de la presión.

Los métodos SPH y MPS ha evolucionado rápidamente debido a su capacidad de reproducir múltiples fenómenos físicos y por su aplicabilidad en problemas ingenieriles (Koshizuka et al., 1998; Edmond y Shao, 2002; Koshizuka et al., 2008; Dalrymple y Rogers, 2006; Gotoh, 2009; Gotoh y Khayyer, 2016; Gotoh y Khayyer, 2018). Por ejemplo, estos métodos se han aplicado a problemas de hidrodinámica marina como la generación de olas en la aproximación cnoindal de la teoría no lineal (Koshizuka et al., 1998; Gotoh et al., 2005; Jaime-Ledezma et al., 2019). Los métodos SPH y MPS también se han aplicado en la teoría no-lineal para la simulación de la propagación y rompimiento de olas solitarias (Ataie-Ashtiani et al., 2006; Sanchez-Mondragon y Vazquez-Hernandez, 2018). Además, se ha aplicado el método MPS acoplado con el modelo de turbulencia SPS-LES a problemas de flujos sobre desniveles (Sahebari et al., 2011) y el método MPS acoplado con el modelo de turbulencia al problema de un generador de olas tipo pistón obteniendo perfiles de ola tipo solitaria y oleaje regular e irregular (Kolahdoozan et al., 2014).

Otro tipo de estudios en los que se han aplicado los modelos SPH y MPS son los relativos a shipping water (agua en cubierta) y sloshing (fluidos agitados en contenedores), los cuales pueden provocar grandes daños estructurales a barcos o plataformas marinas (Kouh et al., 2007; Lee et al., 2011; Shibata et al., 2009; Shibata et al., 2011; Shibata et al., 2012; Sueyoshi et al., 2005).

El comportamiento del fluido simulado es validado comúnmente con predicciones teóricas o con resultados experimentales, en la mayoría de los casos reproduciendo de forma adecuada las propiedades físicas de interés como presión y velocidad del fluido. Aun así, los métodos han presentado algunas dificultades al reproducir con exactitud la presión hidrostática y la presión hidrodinámica teniendo problemas de inestabilidad como oscilaciones numéricas con frecuencias y amplitudes sobrevaluadas en el histórico de la presión de impacto y distribuciones anormales en el campo de la presión (Ataie-Ashtiani y Farhadi, 2006; Khayyer y Gotoh, 2009; Kondo y Koshizuka, 2011; Khayyer y Gotoh, 2011; Lee et al., 2011; Tanaka y Masunaga, 2010; Sanchez-Mondragon, 2016; entre muchos otros trabajos). Estas dificultades y otras relativas a la reducción del tiempo computacional (Gotoh et al., 2005; Zhu et al., 2011) han sido superadas por medio de una serie de mejoras que se abordarán más adelante.

Este trabajo se desarrolla por medio del método MPS debido a que es uno de los modelos Lagrangianos más representativos y a que su desarrollo más reciente, comparado con el método SPH, supone un área abierta para la propuesta de mejoras. En los siguientes capítulos se describirá la formulación del método MPS y sus diferentes versiones (la original del modelo incompresible basado en la densidad de partículas, la basada en la divergencia de la velocidad y la débilmente compresible); asimismo, algunas de las mejoras más importantes propuestas a los modelos de los operadores diferenciales se explicarán brevemente. El método MPS se aplica al problema del rompimiento de presa, dado que es uno de los problemas clásicos para analizar los modelos en la dinámica de fluidos computacional, esto por su propia complejidad. En las simulaciones realizadas se muestran las bondades de los modelos Lagrangianos como herramientas poderosas para modelar problemas hidrodinámicos complejos. 
Para esto, se presentará una comparación entre los resultados de las versiones del método MPS configuradas con las mejoras propuestas que proporcionen una optimización en los resultados.

\section{MÉtodo de movimiento de ParTículas SEMI-IMPlícito}

Las ecuaciones gobernantes del método son las leyes de conservación de masa y momentum; para un fluido viscoso e incompresible, son la ecuación de continuidad y la ecuación de Navier-Stokes, respectivamente:

$$
\begin{gathered}
\frac{D \rho}{D t}=0, \\
\frac{D \vec{u}}{D t}=-\frac{1}{\rho} \nabla P+v \nabla 2 \vec{u}+\vec{F},
\end{gathered}
$$

La ecuación de continuidad está escrita respecto a la densidad $\rho$ para un fluido incompresible. De la ecuación de Navier-Stokes, $\vec{u}$ representa la velocidad, $D / D t$ denota la diferenciación Lagrangiana que incluye los términos de convección; el lado derecho de la ecuación de Navier-Stokes se compone por el gradiente de presión $\nabla P$, el Laplaciano de la velocidad $\nabla 2 \vec{u}$ para el cálculo de la viscosidad (con $v$ la viscosidad cinemática) y $\vec{F}$ fuerzas externas. Es muy importante hacer notar que en la aplicación del método MPS todos los términos expresados por operadores diferenciales deberán ser remplazados por los modelos de interacción de partículas (Koshizuka y Oka, 1996; Koshizuka et al., 1998).

\section{1. Modelos de interacción de partículas}

Las interacciones entre partículas en el método MPS están determinadas por una función núcleo, la cual modela una interacción finita entre partículas y ayuda a la construcción de los operadores diferenciales. A continuación se muestran algunas de las funciones núcleo que han sido usadas en diversos trabajos:

$$
\begin{gathered}
w(r)=\frac{r_{e}}{r}-1, \text { para } 0 \leq r<r_{e} \mathrm{y} 0, \text { para } r_{e} \leq r . \quad \text { Koshizuka } e t a l .(1998) \\
w(r)=e^{-\alpha(r / r e)^{2}}, \text { para } 0 \leq r \leq r_{e} \mathrm{y} 0, \text { para } r_{e}<r . \quad \text { Belytschko } \text { et al. }(1996) \\
w(r)=\left(1-r / r_{e}\right)^{3}\left(1+r / r_{e}\right)^{3} \text {, para } 0 \leq r<r_{e} \mathrm{y} 0, \text { para } r_{e} \leq r . \quad \text { Lee } e t \text { al. }(2011) \\
w(r)=\left(1-r / r_{e}\right)^{3}, \text { para } 0 \leq r<r_{e} \mathrm{y} 0, \text { para } r_{e} \leq r . \quad \text { Shakibaeinia y Jin }(2010) \\
w(r)=\frac{40}{7 \pi r_{e}^{2}}\left(1-6\left(r / r_{e}\right)^{2}+6\left(r / r_{e}\right)^{3}\right), \text { para } 0 \leq r<0.5 r_{e}, w(r)=\frac{10}{7 \pi r_{e}^{2}}\left(2-2\left(\frac{r}{r_{e}}\right)\right)^{3}, \text { para } 0.5 r_{e}<r(7) \\
<r_{e} \mathrm{y} 0, \text { para } r_{e}<r . \quad \text { Shao y Lo }(2003)
\end{gathered}
$$




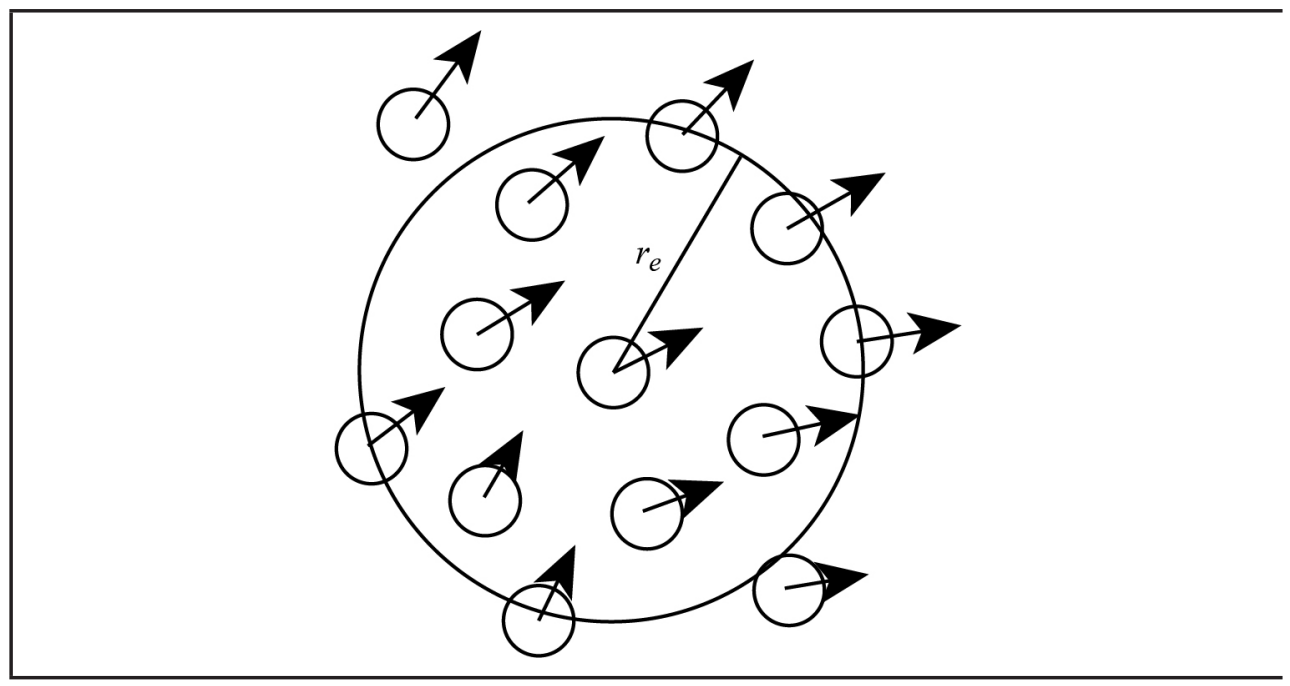

FIGURA 1

Radio de interacción entre partículas

Fuente: elaboración propia.

Estas funciones dependen de $r$, la distancia entre dos partículas $i$ y $j$, y $r_{e}$ el radio de interacción o tamaño del núcleo (kernel size), que determina el radio del área o núcleo de las partículas con las que interactuará la partícula $i$. Lo anterior asegura que partícula $i$ sólo interactúe con un número finito de partículas (figura 1 ).

Dentro de este radio se considera que la función núcleo pondera la interacción con sus vecinos. De esta forma, en los modelos numéricos discretos de los operadores diferenciales se tiene que entre más cercanas se encuentren las partículas mayor será el valor de la función núcleo y por tanto el valor de la interacción será mayor. La formulación de una función núcleo sigue las condiciones propuestas por Gingold y Monaghan (1982):

$$
\begin{gathered}
\lim _{r_{e \rightarrow 0}} w\left(r, r_{e}\right)=\delta(r), \\
\int_{-\infty}^{\infty} w\left(r, r_{e}\right) d r=1 .
\end{gathered}
$$

De la primera condición (ecuación 8) se observa que la función núcleo tiende a considerar una interacción puntual a medida que el radio de interacción tiende a 0 , mientras que la segunda condición supone que la ponderación dentro del radio de interacción es 1 en el dominio determinado por el radio de interacción.

Cuando la partícula $i$ y sus vecinos $j$ se encuentran localizados en $\vec{r}_{i}$ y $\vec{r}_{j}$ respectivamente, la densidad del número de partículas queda definido de la siguiente forma (Koshizuka et al., 1998):

$$
\langle n\rangle_{i}=\sum_{j \neq i} w\left(\left|\overrightarrow{r_{j}}-\overrightarrow{r_{i}}\right|\right),
$$

donde la densidad $\rho_{n}$ en una unidad de volumen se puede aproximar por el número de partículas en el radio de interacción de la siguiente forma (Koshizuka et al., 1998):

$$
\langle\rho\rangle_{i}=\frac{\left\langle\rho_{n}\right\rangle_{i}}{\int w(r) d v},
$$

Asumiendo que todas las partículas tienen la misma masa, la densidad del fluido se podrá definir como $\langle\rho\rangle=$ $m\left\langle\rho_{n}\right\rangle_{i}$ con $m$ la masa de la partícula (Koshizuka et al., 1998). Entonces, se puede considerar que la densidad 
del fluido es proporcional a la densidad del número de partículas. Para flujos incompresibles se requiere que la densidad del fluido sea constante, lo cual es equivalente a que la densidad del número de partículas se mantenga constante $n^{0}$.

\subsection{Operadores diferenciales}

Los operadores diferenciales en el método MPS son modelados a través de las funciones núcleo definidas por Koshizuka y Oka (1996) y Koshizuka et al. (1998) de la siguiente forma: si $\varphi$ es una cantidad física escalar y $\vec{u}$ es una cantidad física vectorial los operadores diferenciales gradiente, divergencia y el Laplaciano pueden ser modelados a través de su interacción entre vecinos por medio de una función de interpolación de la siguiente manera:

$$
\begin{gathered}
\langle\nabla \varphi\rangle_{i}=\frac{d}{n^{0}} \sum_{j \neq i} \frac{\varphi_{j}-\varphi_{i}}{\left|\overrightarrow{r_{j}}-\overrightarrow{r_{i}}\right|}\left(\overrightarrow{r_{j}}-\overrightarrow{r_{i}}\right) w\left(\left|\overrightarrow{r_{j}}-\overrightarrow{r_{i}}\right|\right), \\
\langle\nabla . \vec{u}\rangle_{i}=\frac{d}{n^{0}} \sum_{j \neq i} \frac{\left(\overrightarrow{u_{j}}-\overrightarrow{u_{i}}\right) \cdot\left(\overrightarrow{r_{j}}-\overrightarrow{r_{i}}\right)}{\left|\overrightarrow{r_{j}}-\overrightarrow{r_{i}}\right|} w\left(\left|\overrightarrow{r_{j}}-\overrightarrow{r_{i}}\right|\right), \\
\left\langle\nabla^{2} \varphi\right\rangle_{i}=\frac{2 d}{\lambda n^{0}} \sum_{j \neq i}\left(\varphi_{j}-\varphi_{i}\right) w\left(\left|\overrightarrow{r_{j}}-\overrightarrow{r_{i}}\right|\right),
\end{gathered}
$$

donde $d$ es igual al número de dimensiones espaciales, $n^{0}$ es la densidad de número de partículas inicial y $\lambda$ es un parámetro introducido para mantener la misma tasa de varianza que en las soluciones analíticas y está determinado por la expresión:

$$
\lambda=\frac{\int w(r) r^{2} d v}{\int w(r) d v}
$$

\section{2. 1. Mejoras a los operadores diferenciales}

Para mejorar la estabilidad del método MPS tanto en la distribución de la presión como en la presión de impacto representada a través de la historia de la presión de impacto y la estabilidad de la superficie libre se han propuesto varias mejoras a los modelos. A continuación se describen algunas mejoras al modelo del gradiente de presión:

a) La mejora propuesta por Koshizuka et al. (1998) considera el valor más pequeño de la presión en el radio de interacción $\hat{P}_{i}=\min \left\{P_{i}, P_{j}, \min \right\}$ y lo sustituye por el valor de la presión en la $i$-ésima partícula del modelo del gradiente original (ecuación 12) de la siguiente forma:

$$
\left\langle\frac{1}{\rho} \nabla P\right\rangle_{i}=\frac{d}{\rho_{i} n^{0}} \sum_{j \neq i} \frac{P_{j}-\hat{P}_{i}}{\left|\overrightarrow{r_{j}}-\overrightarrow{r_{i}}\right|^{2}}\left(\overrightarrow{r_{j}}-\overrightarrow{r_{i}}\right) w\left(\left|\overrightarrow{r_{j}}-\overrightarrow{r_{i}}\right|\right) .
$$

Con esta mejora se asegura que la fuerza de presión entre partículas sea únicamente repulsiva, lo que evita el acumulamiento de partículas en determinadas zonas y a su vez evita cambios bruscos en la densidad de número de partículas manteniendo la ecuación de compresibilidad estable. 
b) La mejora propuesta por Lee et al. (2011) considera una interacción repulsiva entre partículas en el gradiente de presión al cambiar el signo en el modelo del gradiente (ecuación 12):

$$
\left\langle\frac{1}{\rho} \nabla P\right\rangle_{i}=\frac{d}{\rho_{i} n^{0}} \sum_{j \neq i} \frac{P_{j}+P_{i}}{\left|\overrightarrow{r_{j}}-\overrightarrow{r_{i}}\right|^{2}}\left(\overrightarrow{r_{j}}-\overrightarrow{r_{i}}\right) w\left(\left|\overrightarrow{r_{j}}-\overrightarrow{r_{i}}\right|\right),
$$

c) La mejora propuesta por Sanchez-Mondragon (2016) considera un término adicional repulsivo entre la partícula $\hat{P}_{i}=\min \left\{P_{i}, P_{j}, \min \right\}$ y la partícula $P_{i}$ :

$$
\begin{aligned}
& -\left\langle\frac{1}{\rho} \nabla P\right\rangle_{i}=-\frac{d}{n^{0}} \sum_{j \neq i}\left[\frac{2}{\rho_{i}+\rho_{j}} \frac{P_{j}-\hat{P}_{i}}{\left|\vec{r}_{j}-\vec{r}_{i}\right|^{2}}\left(\vec{r}_{j}-\vec{r}_{i}\right) w\left(\left|\vec{r}_{j}-\vec{r}_{i}\right|\right)\right] \\
& -\left[\frac{d}{n^{0}} \frac{2}{\rho_{i}+\rho_{\text {jmin }}} \frac{P_{j}-\hat{P}_{i}}{\left|\vec{r}_{j \text { min }}-\vec{r}_{i}\right|}\left(\vec{r}_{j \text { min }}-\vec{r}_{i}\right) w\left(\left|\vec{r}_{j m i n}-\vec{r}_{i}\right|\right)\right],
\end{aligned}
$$

de tal forma que se garantiza que la interacción entre estas partículas sea también repulsiva.

d) Otra variación a la mejora propuesta por Lee et al. (2011) es la de Khayyer y Gotoh (2008), la cual considera un término adicional a segundo orden. La expresión se muestra a continuación:

$$
\left\langle\frac{1}{\rho} \nabla P\right\rangle_{i}=\frac{d}{\rho_{i} n^{0}} \sum_{j \neq i} \frac{P_{j}+P_{i}-\hat{P}_{j}-\hat{P}_{i}}{\left|\overrightarrow{r_{j}}-\overrightarrow{r_{i}}\right|^{2}}\left(\overrightarrow{r_{j}}-\overrightarrow{r_{i}}\right) w\left(\left|\overrightarrow{r_{j}}-\overrightarrow{r_{i}}\right|\right) .
$$

En cuanto al cálculo del operador Laplaciano también se han propuesto algunas mejoras como las siguientes:

a) Khayyer y Gotoh (2010) consideraron una aproximación a la construcción del Laplaciano al aplicar la divergencia al modelo del gradiente. La expresión se muestra a continuación:

$$
\left\langle\nabla^{2} \varphi\right\rangle_{i}=\frac{d}{n^{0}} \sum_{j \neq i}\left(\nabla \varphi_{i j} \cdot \nabla w\left(\left|\vec{r}_{j}-\vec{r}_{i}\right|\right)-\varphi_{i} \nabla^{2} w\left(\left|\vec{r}_{j}-\vec{r}_{i}\right|\right)\right)
$$

b) Xu y Jin 2016, aplicando consideraciones similares a las de Khayyer y Gotoh (2010), desarrollan la siguiente expresión alternativa:

$$
\left\langle\nabla^{2} \varphi\right\rangle_{i}=\frac{d}{n^{0}} \sum_{j \neq i} \frac{\left(\varphi_{j}-\varphi_{i}\right)}{\left|\overrightarrow{r_{j}}-\vec{r}_{i}\right|^{2}}\left[(3 d-2) w\left(\left|\overrightarrow{r_{j}}-\vec{r}_{i}\right|\right)+\left(\overrightarrow{r_{j}}-\overrightarrow{r_{i}}\right) \frac{\partial w\left(\left|\overrightarrow{r_{j}}-\overrightarrow{r_{i}}\right|\right)}{\partial\left(\overrightarrow{r_{j}}-\overrightarrow{r_{i}}\right)}\right],
$$

Cada una de las modificaciones a los modelos de los operadores han demostrado mejoras significativas dependiendo de las aplicaciones propuestas, por lo que en la mayoría de los casos es necesario evaluar cuál es el modelo que mejor representa el fenómeno a simular. 


\section{3. Modelo de incompresibilidad}

El método MPS se desarrolla en dos etapas: en la primera se mueven las partículas usando la ecuación de $\mathrm{Na}$ vier-Stokes sin considerar el término del gradiente de presión y en la segunda se realiza una corrección a través de la aplicación del mismo. Suponiendo que al no agregar el término del gradiente de presión no se cumple la condición de incompresibilidad (no se mantiene el número de partículas constante), entonces la densidad del número de partículas $n^{*}$ se desvía del valor constante $n^{0}$. Se puede considerar que la densidad del número de partículas $n^{*}$ está desviado por un valor $n^{\prime}$ :

$$
n^{*}+n^{\prime}=n^{0}
$$

Esta expresión sugiere que la desviación de la densidad del número de partículas $n^{\prime}$ se debe a la divergencia de la velocidad, lo cual se puede expresar de la siguiente forma:

$$
\frac{n^{\prime}}{n^{0} d t}=-\nabla \cdot \vec{u}
$$

Además, suponiendo que la modificación de la velocidad es derivada del término de la presión de la ecuación de conservación de momentum, se obtiene la expresión:

$$
\vec{u}^{\prime}=-\frac{d t}{\rho} \nabla P^{n+1}
$$

Por último, de las últimas ecuaciones (ecuaciones 23 y 24) se construye la ecuación de Poisson para la presión formulada por Koshizuka y Oka (1996) y Koshizuka et al. (1998):

$$
\left\langle\nabla^{2 P n+1}\right\rangle_{i}=-\frac{\rho_{i}}{d t^{2}} \frac{\left\langle n^{*}\right\rangle_{i}-n^{0}}{n^{0}}
$$

Esta ecuación se discretiza usando el modelo del operador Laplaciano definido por la ecuación (14), con lo cual se obtiene un sistema de ecuaciones lineales acopladas. Al resolver este sistema de ecuaciones se obtiene implícitamente el valor de la presión para cada partícula que compensa la densidad de numero de partículas y el modelo de incompresibilidad.

\section{3. 1. Mejoras al cálculo de la presión}

La estabilidad numérica del método MPS depende ampliamente del cálculo de la presión y de su aplicación mediante el gradiente de la presión (véase Lee et al., 2011). De esta forma, en la ecuación de Poisson de la presión (ecuación 25) se ha identificado que la diferencia en el término fuente entre la densidad de número de partículas $\left\langle n^{*}\right\rangle_{i}$ (calculada en el paso intermedio) y la constante de la densidad de número de partículas $n^{0}$ representa un problema de estabilidad, pues grandes variaciones de esta diferencia provocan presiones muy grandes (en la mayoría de los casos valores no físicos) produciendo inestabilidades. Por lo anterior, se han propuesto las siguientes mejoras: 
a) La mejora propuesta por Khayyer y Gotoh (2009) está formulada con términos de alto orden en el término fuente de la ecuación de Poisson. La expresión se muestra a continuación:

$$
\left\langle\nabla^{2 P n+1}\right\rangle_{i}=-\frac{\rho_{i}}{d t^{2}}\left(\frac{D n}{D t}\right)^{*}=-\frac{\rho_{i}}{d t^{2}} \sum_{j \neq i} \frac{r_{e}}{\left(\vec{r}_{j}-\vec{r}_{i}\right)^{3}}\left(x_{i j} u_{i j}+y_{i j} v_{i j}\right)^{*}
$$

Esta modificación permite considerar la direccionalidad de la diferencia del número de partículas en cada paso temporal.

b) Una mejora a la formulación de la ecuación de Poisson que permite obtener valores más estables de la presión es la propuesta por Tanaka y Masunaga (2010). En esta modificación no se considera como término principal, en el término fuente, a la diferencia de la densidad del número de partículas; en lugar de lo anterior se considera la contribución de la divergencia de la velocidad en el término fuente de la ecuación de Poisson:

$$
\left\langle\nabla^{2 P n+1}\right\rangle_{i}=\frac{\rho_{i}}{d t}\left\langle\nabla \cdot \vec{u}^{*}\right\rangle_{i}-\gamma^{*} \frac{\rho}{d t^{2}} \frac{\langle n\rangle_{i}-n^{0}}{n^{0}}
$$

con $0<\gamma^{*}<1$. La variación a esta formulación fue planteada por Lee et al. (2011) y considera un parámetro extra para la estabilidad numérica. La expresión es la siguiente:

$$
\left\langle\nabla^{2 P n+1}\right\rangle_{i}=\left(1-\gamma^{*}\right) \frac{\rho_{i}}{d t}\left\langle\nabla \cdot \vec{u}^{*}\right\rangle_{i}-\gamma^{*} \frac{\rho}{d t} \frac{\langle n\rangle_{i}-n^{0}}{n^{0}}
$$

con $0.01<\gamma^{*}<0.05$. La ecuación 28, además de mejorar la estabilidad en el cálculo de la presión, supone la reducción de un paso en el algoritmo original del método MPS dado que no requiere actualizar la posición de las partículas en cada paso temporal.

De forma alternativa al cálculo de las presiones usando la ecuación de Poisson en el método MPS, se pude hacer uso de la ecuación de estado propuesta por Shakibaeinia y Jin (2010) por medio de la siguiente expresión:

$$
\left\langle P^{n+1}\right\rangle_{i}=\frac{\rho_{i} c_{0}^{2}}{\gamma}\left(\left(\frac{n_{i}^{*}}{n^{0}}\right)^{\gamma}-1\right)
$$

donde $c_{0}$ es la velocidad del sonido y el parámetro $\gamma=7$.

c) Otra versión al modelo de incompresibilidad la constituye la mejora propuesta al parámetro $\lambda$ del operador Laplaciano (ecuación 15) aplicado a resolver la ecuación de Poisson. Para esto, Sanchez-Mondragon (2016) consideró la amortiguación de las variaciones de la posición usando las variaciones de la velocidad en el paso intermedio:

$$
\lambda_{i}^{*}=\frac{\int w(r) r^{2} d v}{\int w(r) d v}-\frac{\int w(r) u^{2} d v}{\int w(r) d v}(d t)^{2}
$$

Esta ecuación presentó mejoras en el cálculo numérico del histórico de presión de impacto tanto en la ecuación de Poisson de la ecuación (22), en Sanchez-Mondragon (2016), como en la ecuación de Poisson basada en la divergencia (ecuación 24), en Sanchez-Mondragon y Vazquez-Hernandez (2018) y Jaime-Ledezma et al. (2019). 


\subsection{Condición en la frontera}

La condición de frontera determinada por Koshizuka y Oka (1996) y Koshizuka et al. (1998) que resuelve el sistema de ecuaciones implícito en la ecuación de Poisson para la presión corresponde a asignar el valor de la presión atmosférica o 0 a la presión de las partículas en la superficie libre, las cuales se definen como partículas cuya densidad de número de partículas es menor que un porcentaje de la densidad de número de partículas inicial. Esta condición se expresa de la siguiente forma:

$$
\langle n\rangle_{i} \leq \beta n^{0} .
$$

El parámetro considera valores entre $0.8 \leq \beta \leq 0.99$, en este trabajo $\beta=0.97$.

En cuanto a los objetos sólidos con los que interactúa el fluido, éstos suelen modelarse usando partículas que tienen asignadas el valor correspondiente a la densidad del material. Para evitar grandes diferencias en la densidad del número de partículas en dichas fronteras se colocan una o dos capas de partículas de la pared y una o dos capas de "partículas falsas". De ellas, sólo las de la pared se incluyen en el cálculo de la presión y las falsas contribuyen únicamente en el cálculo de la densidad de número de partículas.

\section{5. Formulación del algoritmo del método MPS}

La aplicación del método MPS está basada en la condición de incompresibilidad y está formulada a través de un algoritmo de predicción-corrección (Koshizuka et al., 1998), de tal manera que las velocidades y posiciones se calculan por medio de la forma discreta de las ecuaciones de Navier-Stokes, las cuales se resuelven para cada instante de tiempo y, en un proceso iterativo dependiente del intervalo de tiempo $d t$, se consideran las siguientes ecuaciones iterativas para la actualización de la velocidad y la posición:

$$
\begin{gathered}
\vec{u}_{i}^{n+1}=\vec{u}_{i}^{n}+\vec{u}_{i}^{\prime}+d t\left(-\frac{1}{\rho} \nabla P_{i}+v \nabla 2 \overrightarrow{u_{i}}+\vec{g}_{i}\right)^{n}, \\
\vec{r}_{i}^{n+1}=\vec{r}_{i}^{n}+\vec{u}_{i}^{n+1} d t .
\end{gathered}
$$

En la etapa de predicción se considera el cálculo explícito de la contribución de la viscosidad y fuerzas externas en la forma discreta de la ecuación de Navier-Stokes (ecuación 2). Posteriormente, de las expresiones para calcular la presión se construyen los algoritmos para las diferentes versiones del método MPS. En la figura 2 se observan los diagramas de flujo de las tres versiones del método: la ecuación de Poisson (ecuación 25) da lugar al algoritmo original del método MPS, de las ecuaciones 27 y 28 se desarrolla el algoritmo del método MPS basado en la divergencia de la velocidad, el cual se diferencia del algoritmo del método original dado que el término fuente de la ecuación de Poisson no depende de los valores intermedios, por ello no es necesario repetir el paso de búsqueda de vecinos (Tanaka y Masunaga, 2010) al reducir la cantidad de cálculos computacionales respecto a la formulación original del algoritmo. Por último, de la ecuación 29 se desarrolla el algoritmo del método débilmente compresible con lo cual se reduce el tiempo de cómputo al no ser necesario resolver el sistema de ecuaciones de la ecuación de Poisson para calcular el valor de la presión en dada partícula del fluido (Shakibaeinia y Jin, 2010).

En cuanto a la elección del paso temporal que permita la convergencia de las soluciones, se considera el número de Courant, el cual implica que el intervalo de tiempo no debe ser mayor que la relación entre la velocidad del fluido y el tamaño de la celda o el tamaño de la partícula, i. e., $\frac{v}{\Delta x} \Delta t \leq C$, con $C$ el número de Courant $(C \leq 1)$. Lo anterior implica que la información de la partícula no debe pasar a otra sin interactuar con su vecino más cercano. 


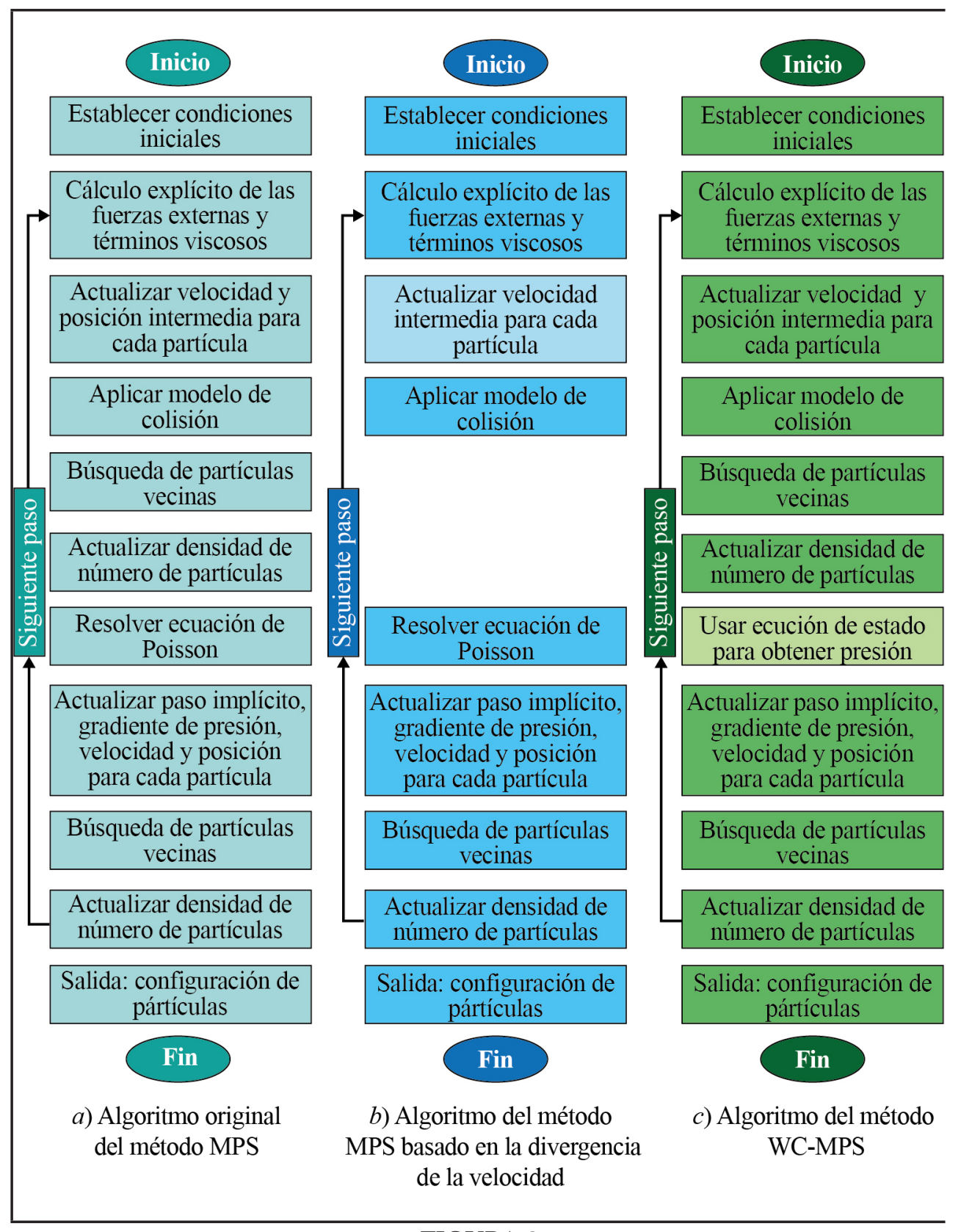

\section{FIGURA 2}

Diagrama de flujo correspondiente a los modelos del método MPS

Fuente: elaboración propia.

\subsection{Modelos de turbulencia}

En el estudio de hidrodinámica de fluidos es importante el modelado de fenómenos turbulentos, los cuales se presentan en flujos con números de Reynolds altos, considerando la contribución del tensor de esfuerzos de Reynolds $(\vec{\tau})$ en la ecuación de Navier-Stokes como sigue:

$$
\frac{D \vec{u}}{D t}=-\frac{1}{\rho} \nabla P+v \nabla 2 \vec{u}+\frac{1}{\rho} \nabla \cdot \vec{\tau}+\vec{F}
$$


Para calcular esta contribución es necesario modelar este fenómeno; de la aproximación con escala de sub-partícula (SPS: Sub Particle Scale), la viscosidad cinemática turbulenta se modela por medio de la siguiente expresión:

$$
\frac{\tau_{i j}}{\rho_{i}}=2 v_{t} S_{i j}-\frac{2}{3} k \delta_{i j}
$$

donde $v_{t}$ es la viscosidad cinemática del remolino, $k$ es la energía cinética turbulenta, $\delta_{i j}$ es la delta de Kronecker y $\delta_{i j}$ es la componente del tensor de estrés y está definida por la siguiente expresión:

$$
S_{i j}=\frac{1}{2}\left(\frac{\partial u_{i}}{\partial x_{j}}+\frac{\partial u_{j}}{\partial x_{i}}\right)
$$

a) Usando la aproximación formulada por Smagorinski (1963), la viscosidad cinemática se puede calcular con el modelo SPS de simulación de grandes remolinos (SPS-LES: Large Eddy Simulation) como:

$$
v_{t}=\left(C_{S} \Delta l\right)^{2}|S|, \text { con }|S|=\left(2 S_{i j} S_{i j}\right)^{1 / 2}
$$

con la constante $C_{S}=0.15$ usando la formulación de Gotoh et al. (2001), modelo que ha sido aplicado en trabajos como el de Sahebari et al. (2011).

b) Usando el modelo de turbulencia $k-\varepsilon$ la viscosidad cinemática se puede determinar a través de la formulación propuesta por Shao (2006) como sigue:

$$
v_{t}=C_{d} \frac{k^{2}}{\varepsilon}, \ldots
$$

con $C_{d}$ una constante empírica, $C_{d}=0.09$. La energía cinética turbulenta, $k$ y la tasa de disipación de energía turbulenta, $\varepsilon$, se obtienen resolviendo las siguientes ecuaciones acopladas:

$$
\begin{gathered}
\frac{D k}{D t}=\nabla \cdot\left(\frac{v t}{\sigma k} \nabla k\right)+P_{k}-\varepsilon, \\
\frac{D \varepsilon}{D t}=\nabla \cdot\left(\frac{v_{t}}{\sigma_{\varepsilon}} \nabla \varepsilon\right)+c_{1 \varepsilon} \frac{\varepsilon}{k} P_{k}-c_{2 \varepsilon} \frac{\varepsilon^{2}}{k}
\end{gathered}
$$

con $\sigma_{k}=1.0, \sigma_{\varepsilon}=1.3, c_{1 \varepsilon}=1.44$ y $c_{2 \varepsilon}=1.92$ constantes empíricas y $P_{k}$ la tasa de producción de turbulencia (Shao, 2006). En dos dimensiones y coordenadas cartesianas $P_{k}$ está definido como (Shao, 2006):

$$
P_{k}=v_{t}\left[2\left(\frac{\partial u}{\partial x}\right)^{2}+2\left(\frac{\partial v}{\partial y}\right)^{2}+\left(\frac{\partial u}{\partial y}+\frac{\partial v}{\partial x}\right)^{2}\right]
$$

donde la divergencia del tensor de estrés se tiene que resolver para cada intervalo de tiempo independientemente del modelo de turbulencia usado.

\section{EJEMPLOS DEL MÉTODo MPS}

Los ejemplos del método MPS en sus tres versiones fueron desarrollados usando los algoritmos de la figura 2. Las condiciones iniciales del rompimiento de presa mostradas en la gráfica 1 fueron usadas por Kondo y Koshizuka (2011) y Sanchez-Mondragon (2016) para probar las mejoras propuestas en los respectivos trabajos. 
En todas las simulaciones se usaron dos capas de partículas en la pared y dos capas de partículas falsas, mostradas en verde y rojo respectivamente. Los parámetros físicos usados son los siguientes: densidad del agua 1000 $\mathrm{kg} / \mathrm{m}^{3}$, constante de gravedad $g=9.81 \mathrm{~m} / \mathrm{s}^{2}$ y viscosidad cinemática $1.028 \times 10^{-6 \mathrm{~m}^{2}} / \mathrm{s}$. La distancia inicial entre las partículas es de $0.012 \mathrm{~m}$, el paso temporal es fijo, $0.0014 \mathrm{~s}$ en las versiones original y basado en la divergencia, mientras que para el método débilmente compresible 0.0004 s. La elección de los valores del paso temporal toma en consideración el número de Courant y una serie de pruebas en las que se elige el máximo valor del paso temporal que mantenga la simulación estable (resultados de las pruebas no mostrados para cumplir con las restricciones del tamaño de la publicación).

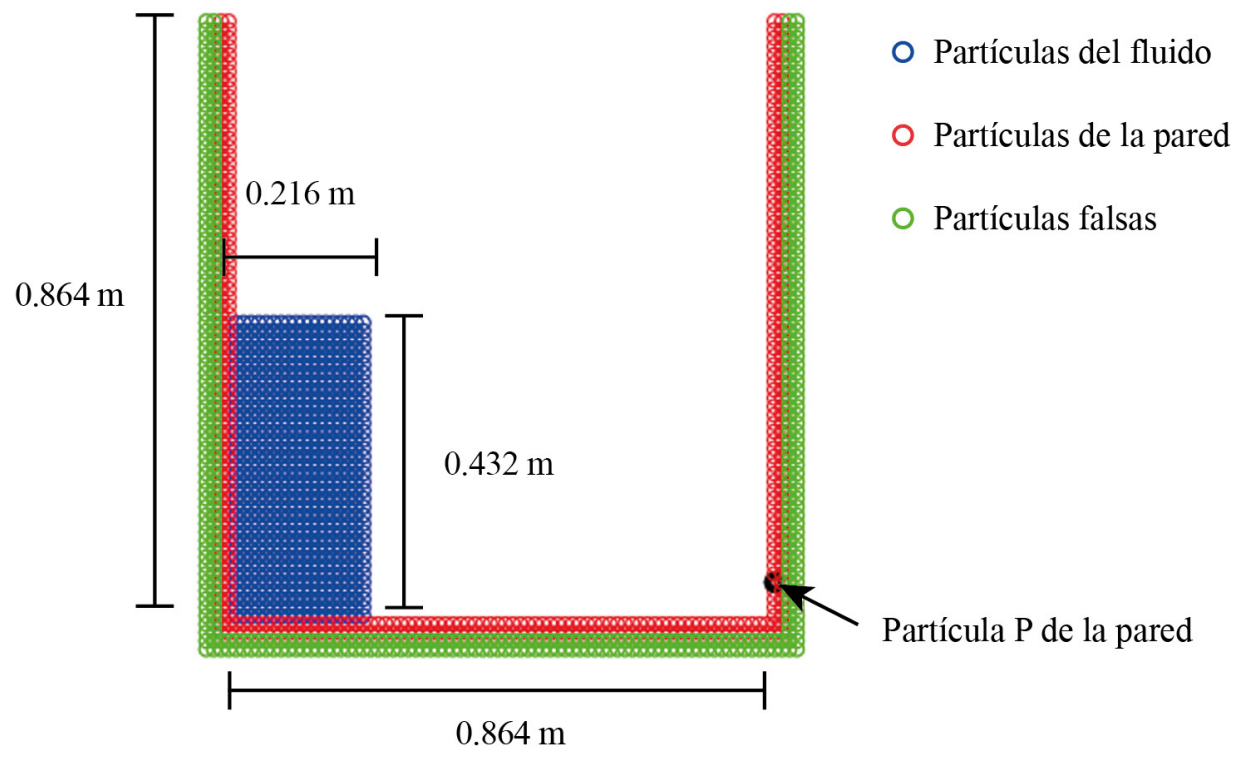

GRÁFICA 1

Condiciones iniciales del problema de rompimiento de presa.

Fuente: elaboración propia.

El caso simulado con el método MPS configurado con el algoritmo original considera el gradiente de la presión calculado con la ecuación 18, la ecuación de Poisson (ecuación 25), con la corrección del parámetro $\lambda *$ de la ecuación 30, el modelo del Laplaciano para el cálculo de la velocidad (ecuación 14) y la función núcleo definida por la ecuación 3 - cabe señalar que en este caso los resultados difieren de los publicados en Sanchez-Mondragon (2016) en el valor considerado de la viscosidad cinemática-. Los resultados de la distribución espacial del campo de presiones para una serie de tiempo se muestra en la gráfica 2, los cuales presentan una distribución de presiones estable, i. e., con capas bien definidas en estratos y una superficie suave en las zonas donde no hay rompimiento, por ejemplo en las dos primeras capturas de la gráfica 2; con esto se concluye que la distribución de presiones y la superficie simulada son estables.

El caso simulado con el método MPS basado en la divergencia de la velocidad considera el gradiente de la presión calculado con la ecuación 18, la ecuación de Poisson con la corrección de la divergencia de la velocidad (ecuación 28), la corrección del parámetro de la ecuación 30 y el modelo del Laplaciano para el cálculo de la velocidad (ecuación 14) y la función núcleo definida por la ecuación 5. La gráfica 3 muestra los resultados de la distribución espacial del campo de presiones para una serie de tiempo. Estos resultados evidencian una distribución de presiones estable, pues el campo de presiones presenta una distribución bien definida y una superficie cuasiestable debido a que se tiene una superficie con algunas rugosidades y en algunos casos salen disparadas partículas fuera de la superficie libre sin coincidir con el comportamiento real, ya que la superficie mostrada en las dos primeras capturas tiene algunas irregularidades (comparar con las respectivas capturas de la gráfica 3). 

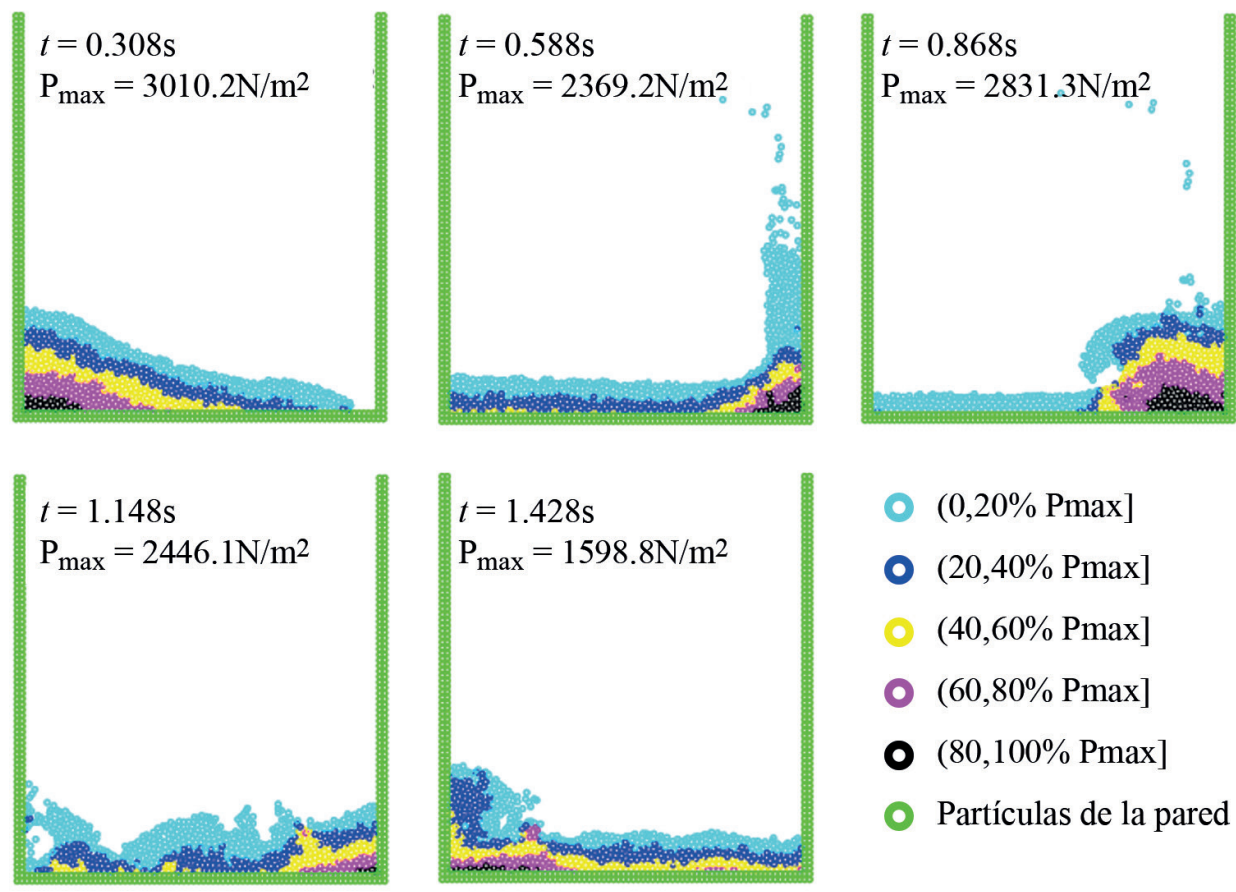
(0,20\% Pmax]
- $(20,40 \%$ Pmax]
- $(40,60 \% \operatorname{Pmax}]$
- $(60,80 \%$ Pmax]
- $(80,100 \%$ Pmax]

- Partículas de la pared

\section{GRÁFICA 2}

Distribución de la presión en el desarrollo de la simulación usando el algoritmo del modelo MPS

basado en la divergencia

Fuente: elaboración propia.

Nota: en la figura se observa la evolución del rompimiento de presa en varias etapas con una distribución de presión en función de la presión máxima (Pmax).
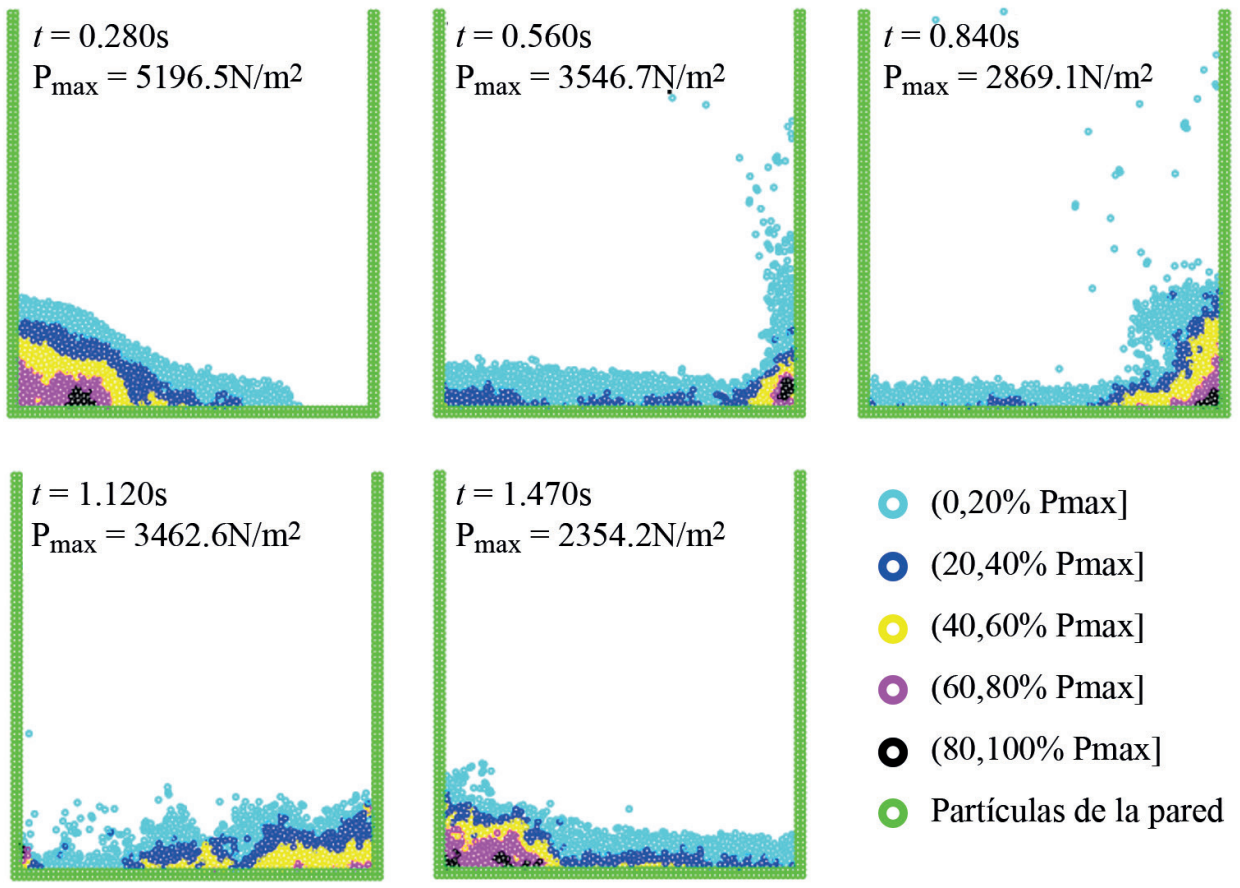

$$
(0,20 \% \text { Pmax] }
$$

- $(20,40 \% \operatorname{Pmax}]$

- $(40,60 \% \operatorname{Pmax}]$

- $(60,80 \%$ Pmax $]$

- $(80,100 \%$ Pmax $]$

- Partículas de la pared

\section{GRÁFICA 3}

Distribución de la presión en el desarrollo de la simulación usando el algoritmo del modelo MPS

basado en la divergencia

Fuente: elaboración propia.

Nota: en la figura se observa la evolución del rompimiento de presa en varias etapas con una distribución de presión en función de la presión máxima (Pmax). 

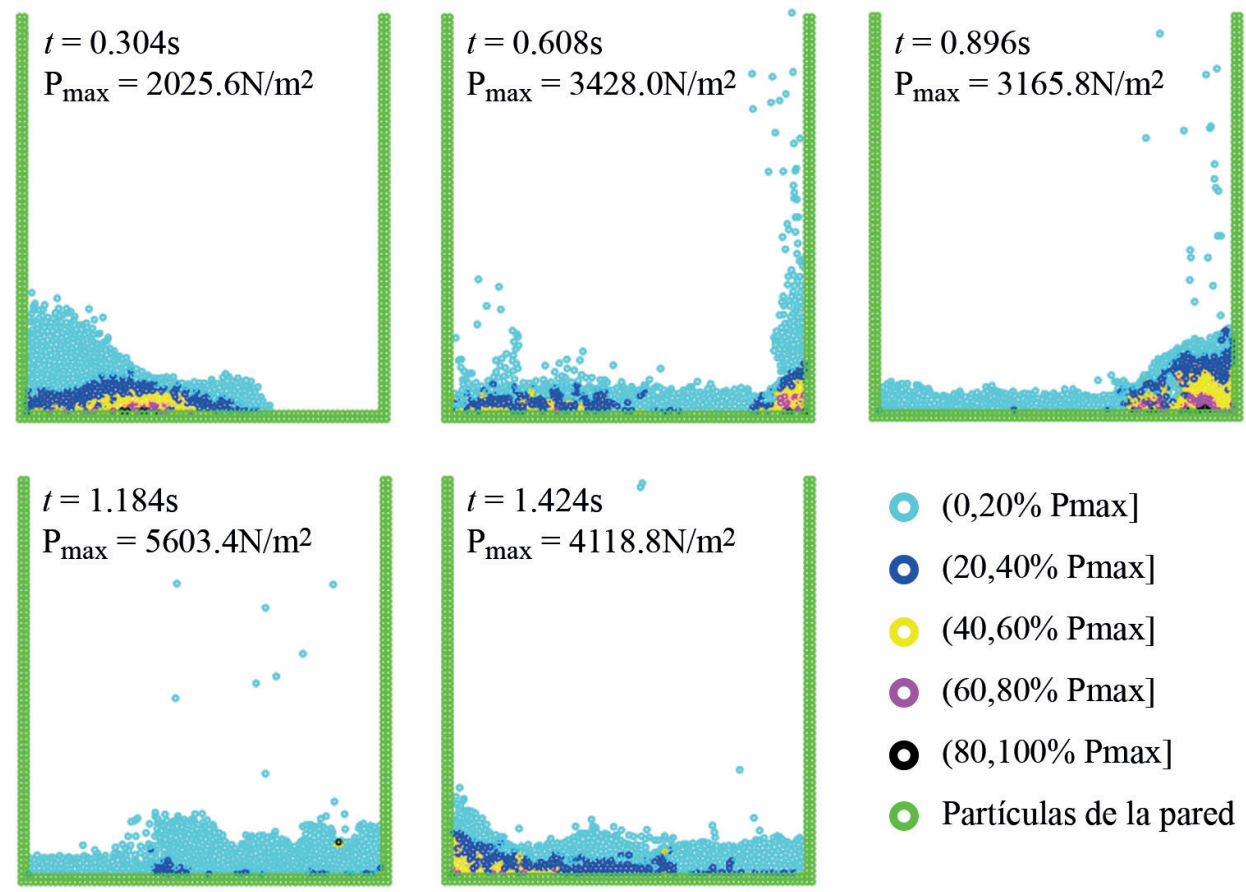

(0,20\% Pmax]

- $(20,40 \%$ Pmax]

(40,60\% Pmax]

- $(60,80 \%$ Pmax]

- $(80,100 \%$ Pmax $]$

- Partículas de la pared

GRÁFICA 4

Distribución de la presión en el desarrollo de la simulación usando el algoritmo del modelo WC-MPS

Fuente: elaboración propia.

Nota: en la figura se observa la evolución del rompimiento de presa en varias etapas con una distribución de presión en función de la presión máxima (Pmax).

El caso simulado con el método WC-MPS considera el algoritmo propio del método basado en la ecuación de estado para el cálculo de la presión (ecuación 29), el gradiente de la presión calculado con la ecuación 18) y el modelo del Laplaciano para el cálculo de la velocidad (ecuación 21), la función núcleo definida por la ecuación 6 y se usó el modelo de turbulencia SPS-LES (ecuaciones 35-37). La gráfica 4 ilustra los resultados de la distribución espacial del campo de presiones para una serie de tiempo. Estos resultados muestran una distribución de presiones y superficie cuasiestable, dado que las distribuciones de presiones no son uniformes (como se puede comparar con las distribuciones de presión en las gráficas 2 y 3 ), además de que en la superficie existen partículas libres con comportamientos inestables provocando una superficie libre con rugosidades.

En la gráfica 5 se muestra el histórico de la presión de impacto en la partícula de la pared $P$, expuesta en la gráfica 1. Este histórico de la presión se obtiene del cálculo numérico en cada paso temporal de la respectiva simulación, pues al ser consideradas como partículas las paredes del contenedor también se resuelven estos valores. Se puede observar que el comportamiento de las presiones en el método MPS con la formulación original (con base en la configuración de Sanchez-Mondragon, 2016) y con la formulación basada en la divergencia de la velocidad (con base en la configuración de Sanchez-Mondragon y Vazquez-Hernandez, 2018) es semejante tanto en magnitud como en la aparición temporal de los picos máximos de la presión; sin embargo, la presión de impacto calculada con la versión del método débilmente compresible (WC-MPS) muestra un desfase en la aparición del primer pico y valores menores de la presión de impacto en todo el histórico de la presión respecto a las otras simulaciones. Este amortiguamiento puede ser provocado por la distribución inestable de las presiones. 


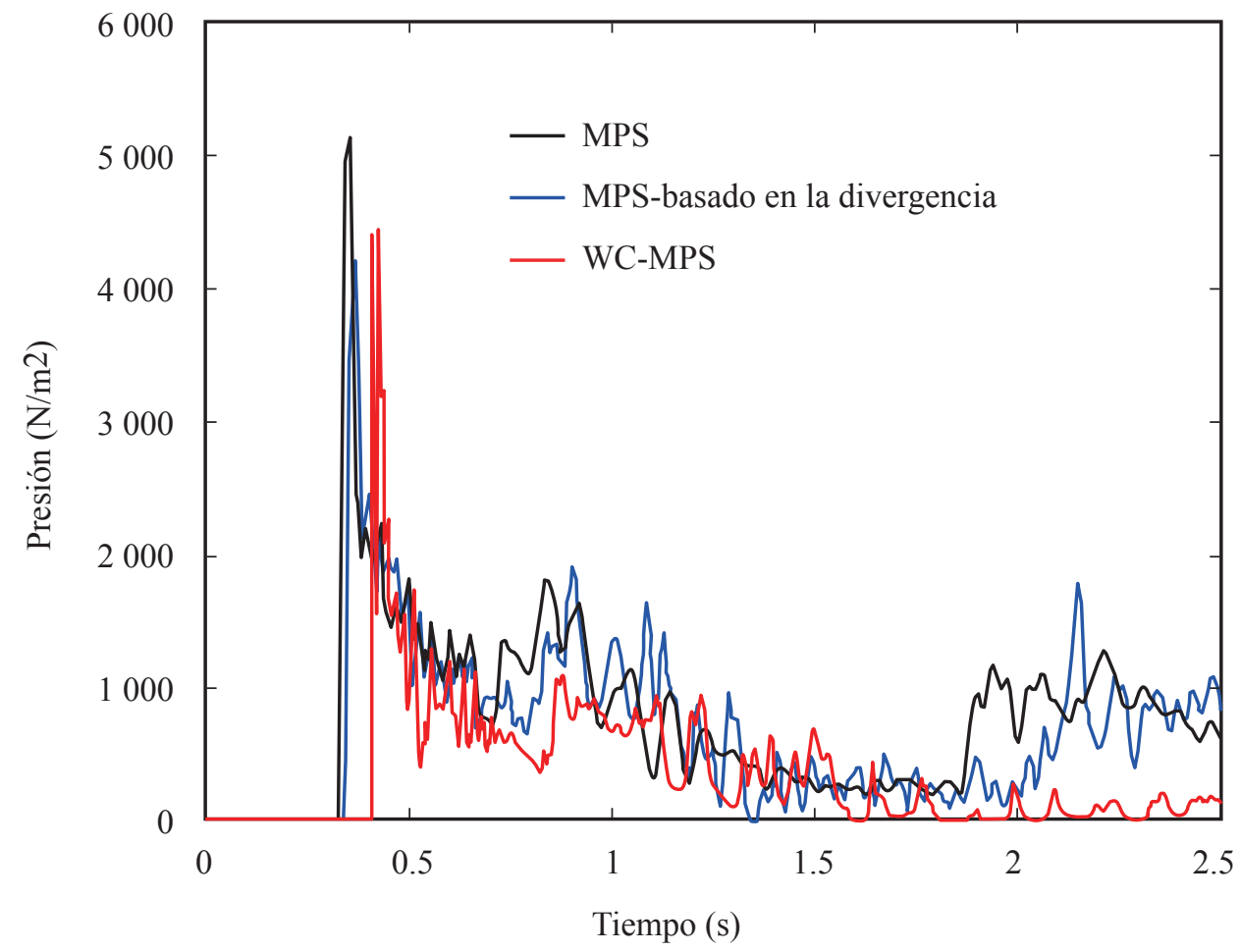

GRÁFICA 5

Historia de la presión de impacto para las tres versiones del modelo MPS simulados Fuente: elaboración propia.

\section{AnÁlisis Prospectivo}

El desarrollo de la dinámica computacional de fluidos es un área de la ciencia y tecnología con un potencial de desarrollo importante con aplicaciones directas en la industria, en particular el método de movimiento de partículas semi-implícito tiene la capacidad de simular con precisión fenómenos no lineales como la formación y rompimiento de oleaje, de gran importancia en el área de la hidrodinámica marina. Actualmente, debido a su reciente creación, el método MPS representa un área abierta para propuestas y mejoras, además de la proyección que tiene su aplicación en tres dimensiones para la simulación de fluidos en medios porosos, así como de mezcla. En particular, el artículo presenta una descripción de las distintas versiones del modelo con una comparación práctica, lo que permitirá introducir al lector en el área de simulación de fluidos usando métodos Lagrangianos.

\section{Conclusiones}

La ecuación de Navier-Stokes se puede resolver numéricamente para problemas de hidrodinámica usando métodos Lagrangianos, en particular el método MPS y sus versiones descritas en este artículo tienen una gran cantidad de aplicaciones. Como ejemplo se aplicaron las tres versiones del modelo con configuraciones especiales al problema del rompimiento de presa, el cual es un problema usado para la comprobación de modelos en el área de la dinámica de fluidos computacional.

La representación extrema en problemas altamente no lineales es ejemplificada en el problema aplicado del rompimiento de presa, el cual se sale de la representación clásica de las herramientas numéricas. En los resultados obtenidos se muestra que el comportamiento general del fluido es muy similar en las simulaciones desarrolladas con la formulación original y con la que es basada en la divergencia, siendo el menos estable la formulación del método débilmente compresible (WC-MPS). Como se describió en la sección 1. 5., el método débilmente com- 
presible requiere de una menor cantidad de cálculos computacionales; sin embargo, como se ha observado en los resultados reportados tiene algunos problemas de estabilidad. Por lo anterior, se considera que la mejora de la estabilidad del método débilmente compresible es un problema abierto. En cuanto a la aplicación del método en sus versiones original y basada en la divergencia, es viable su aplicación, dada la estabilidad que poseen.

A través de la revisión del método MPS se ha podido observar que los modelos Lagrangianos tienen la capacidad de resolver problemas hidrodinámicos complejos, siendo imprescindible revisar las propiedades de cada una de sus versiones para identificar su estabilidad. Aunque este trabajo se ha enfocado en el estudio de las versiones del método MPS, es necesario considerar las diferentes versiones método SPH en futuros trabajos y desarrollar comparaciones, dado que el primero es un modelo más desarrollado.

El desarrollo de pruebas numéricas son temas en auge en la mecánica de fluidos debido a que las simulaciones proveen una solución dinámica del comportamiento de un fluido a través de su descripción matemática a un bajo costo. Con esto en cuenta, la aplicación del método MPS y su extensión a problemas en tres dimensiones constituye una línea de investigación con gran potencial de desarrollo.

\section{Agradecimientos}

Se agradece a la Dirección de Cátedras CONACYT por el apoyo financiero otorgado durante la elaboración de este trabajo.

\section{REFERENCIAS}

Ataie-Ashtiani, B., \& Farhadi, L. (2006). A stable moving-particle semi implicit method for free surfaces flows. Fluid Dyn. Res., 38, 241-256.

Ataie-Ashtiani, B., Shobeyri, G., \& Farhadi, L. (2006). Modified incompressible SPH method for simulating free surface problems. Fluid Dyn. Res., 40(9), 637-661.

Belytschko, T., Krongauz, Y., Organ, D., Fleming, M., \& Krysl, P. (1996). Meshless methods: An overview and recent developments. Comput. Methods Appl. Mech. Eng., 139, 3-47.

Dalrymple, R. A., \& Rogers, B. D. (2006). Numerical modeling of water waves with the SPH method. Coast. Eng., 53(2), 141-147.

Edmond, Y. M. L., \& Shao, S. (2002). Simulation of near-shore solitary wave mechanics by an incompressible SPH method. App. Ocean Res., 24(5), 275-286.

Gingold, R. A., \& Monaghan, J. J. (1977). Smoothed particle hydrodynamics-Theory and application to non-spherical stars. Mon. Not. R. Astron. Soc., 181, 375-389.

Gingold, R. A., \& Monaghan, J. J. (1982). Kernel estimates as a basis for general particle methods in hydrodynamics. J. Comput. Phys., 46, 429-453.

Gotoh, H., (2009). Lagrangian particle method as advanced technology for numerical wave flume. Int.J. Offshore Polar Eng., 19(3), 161-167.

Gotoh, H., Ikari, H., Memita, T., \& Sakai, T. (2005). Lagrangian particle method of wave overtopping on a vertical seawall. Coastal Eng. J., 47, 157-181.

Gotoh, H., \& Khayyer, A. (2016). Current achievements and future perspectives for projection-based particle methods with applications in ocean engineering. J. Ocean Eng. Mar. Energy, 2(3), 251-278. 
Gotoh, H., \& Khayyer, A. (2018). On the state-of-the-art of particle methods for coastal and ocean engineering. Coastal Eng. J., 60(1), 79-103.

Gotoh, H., Shibahara, T., \& Sakai, T. (2001). Sub-particle-scale turbulence model for the MPS Method-Lagrangian flow model for hydraulic engineering. Comput. Fluid Dyn. J., 9, 339-347.

Jaime-Ledezma, L. E., Sanchez-Mondragon, J., Vazquez-Hernandez, A. O., Morales-Viscaya, J. A., \& Ochoa-Ruiz, G. (2019). Simulation of breaking waves on slop beaches integrating the MPS method into Iwagaki wave theory. J. Braz. Soc. Mech. Sci. Eng., 41(170).

Khayyer, A., \& Gotoh, H. (2008). Development of CMPS method for accurate water-surface tracking in breaking waves. Coastal Eng.J., 50(2), 179-207.

Khayyer, A., \& Gotoh, H. (2009). Modified Moving Particle Semi-implicit methods for the prediction of 2D wave impact pressure. Coastal Eng., 56, 419-440.

Khayyer, A., \& Gotoh, H. (2010). A higher order Laplacian model for enhancement and stabilization of pressure calculation by the MPS method. Appl. Ocean Res., 32, 124-131.

Khayyer, A., \& Gotoh, H. (2011). Enhancement of stability and accuracy of the moving particle semi-implicit method.J. Comput. Phys., 230, 3093-3118.

Kolahdoozan, M., Ahadi, M. S., \& Shirazpoor, S. (2014). Effect of turbulence closer models on the accuracy of MPS method for the viscous free surface flow. Sci. Iran., 21(4), 1217-1230

Kondo, M., \& Koshizuka, S. (2011). Improvement of stability in Moving Particle Semi-implicit method. Int. J. Numer. Meth. Fluids., 65, 638-654.

Koshizuka, S., Nobe, A., \& Oka, Y. (1998). Numerical analysis of breaking waves using the moving particle semi-implicit method. Int. J. Numer. Meth. Fluids., 26, 751-769.

Koshizuka, S., \& Oka, Y. (1996). Moving-particle semi-implicit method for fragmentation of incompressible fluid. Nucl. Sci. Eng., 123, 421-434.

Koshizuka, S., Suzuki, Y., \& Harada, T. (2008). Multi-physics simulation for micro fluidic devices using Moving Particle Semi-implicit method. WCCM8 and ECCOMAS 2008, June 30-July 5, Venice.

Kouh, J. S., Chien, H. P., Chang, C. C., \& Chen Y. J. (2007). Simulation of a ship with partially filled tanks rolling in waves by applying Moving Particle Semi-implicit method. International Conference on Engineering Education, September 3-September 7.

Lee, B. H., Park, J. C., Kim, M. H., \& Hwang, S. C. (2011). Step-by-step improvement of MPS method in simulating violent free-surface motions and impact-loads. Comput. Methods Appl. Mech. Eng., 200, 1113 1125.

Lucy, L. B. (1977). A numerical approach to the testing of the fission hypothesis. Astron. J., 82, 1013-1024.

Sahebari, A. J., Jin, Y. C., \& Shakibaeinia, A. (2011). Flow over sills by the MPS mesh-free particle method. J. Hydraul. Res., 49(5), 649-656.

Sanchez-Mondragon, J. (2016). On the stabilization of unphysical pressure oscillations in MPS method simulations. Int. J. Numer. Methods Fluids, 82, 471-492.

Sanchez-Mondragon, J., \& Vazquez-Hernandez, A. O. (2018). Solitary wave collisions by double-dam-broken simulations with the MPS method. Eng. Comput., 35(1), 53-70.

Shakibaeinia, A., \& Jin, Y. C. (2010). A weakly compressible MPS method for modeling of open-boundary free-surface flow. Int. J. Numer. Methods Fluids, 63(10), 1208-1232.

Shao, S., (2006). Simulation of breaking wave by SPH method coupled with model. J Hydraul. Res., 44(3), 338-349. 
Shao, S. D., \& Lo, E. Y. M. (2003). Incompressible SPH method for simulating Newtonian and non-Newtonian flows with a free surface. Adv. Water Resour., 26(7), 787-800.

Shibata, K., Koshizuka, S., Sakai, M., \& Tanizawa, K. (2012). Lagrangian simulations of ship-wave interactions in rough seas. Ocean Eng., 42, 13-25.

Shibata, K., Koshizuka, S., Sakai, M., \& Tanizawa, K. (2011). Transparent boundary condition for simulating nonlinear water waves by a particle method. Ocean Eng., 38, 1839-1848.

Shibata, K., Koshizuka, S., \& Tanizawa, K. (2009). Three-dimensional numerical analysis of shipping water onto a moving ship using a particle method. J. Mar. Sci. Technol., 14, 214-227.

Sueyoshi, M., Zdravko, R., Kishev, R., \& Kashiwagi, M. (2005). A particle method for inmpulsive loads caused by violent sloshing. Abstract for the 20th Int. Workshop on water waves and floating bodies, Spitsbergen (Norway), 29th May- 1st June.

Tanaka, M., \& Masunaga, T., (2010). Stabilization and smoothing of pressure in MPS method by Quasy-Compressibility. J. Comput. Phys., 229, 4279-4290.

$\mathrm{Xu}, \mathrm{T} .$, \& Jin, Y. C. (2016). Improvements for accuracy and stability in a weakly-compressible particle method. Comput. Fluids, 137, 1-14.

Zhu, X. S., Cheng, L., Lu, L., \& Teng, B. (2011). Implementation of the moving particle semi-implicit method on GPU. Science China, 54(3), 523-532.

\section{BY-NC-ND}

